Journal of Mathematics and Informatics

Vol. 11, 2017, 119-123

ISSN: 2349-0632 (P), 2349-0640 (online)

Published 11 December 2017

www.researchmathsci.org

DOI: http://dx.doi.org/10.22457/jmi.v11a15

Journal of

Mathematics and

Informatics

\title{
Special Dio 3-tuples for Pentatope Number
}

\section{G.Janaki and C.Saranya}

Department of Mathematics, Cauvery College for Women,

Trichy-18, Tamilnadu, India.

Received 6 November 2017; accepted 9 December 2017

Abstract. We search for three distinct polynomials with integer coefficients such that the product of any two members of the set added with their sum and increased by a non-zero integer (or polynomial with integer coefficients) is a perfect square.

Keywords: Dio 3-tuples, Pentatope number, polynomials.

AMS Mathematics Subject Classification (2010): 11D25

\section{Introduction}

Mathematics is the language of patterns and relationships, and is used to describe anything that can be quantified. The main goal of Number theory is to discover interesting and unexpected relationships. It is devoted primarily to the study of natural numbers and integers. In [1-7], theory of numbers were discussed. Many mathematicians considered the problem of the existence of Diophantine triples \& special dio 3-tuples with the property $D(n)$ for any arbitrary integer $n$ and also for any linear polynomials $n$ [8-10].

In this communication, we present a few special dio 3-tuples for Pentatope numbers of different ranks with their corresponding properties.

\section{Notation}

$$
\mathrm{PT}_{n}=\text { Pentatope number of } \operatorname{rank} n=\frac{1}{24} n(n+1)(n+2)(n+3)
$$

\section{Basic definition}

A set of three distinct polynomials with integer coefficients $\left(a_{1}, a_{2}, a_{3}\right)$ is said to be a special dio 3-tuple with property $D(n)$ if $a_{i} * a_{j}+\left(a_{i}+a_{j}\right)+n$ is a perfect square for all $1 \leq i<j \leq 3$, where $n$ may be non-zero integer or polynomial with integer coefficients.

\section{Method of analysis}

Case 1: Construction of Dio 3-tuples for Pentatope number of rank $n$ and $n-2$.

Let $a=24 \mathrm{PT}_{n}, \quad b=24 \mathrm{PT}_{n-2}$ be Pentatope numbers of rank $n$ and $n-2$ respectively such that $a b+(a+b)+1$ is a perfect square say $\alpha^{2}$. 


\section{G.Janaki and C.Saranya}

Let $c$ be any non-zero integer such that

$$
\begin{aligned}
& a c+(a+c)+1=\beta^{2} \\
& b c+(b+c)+1=\gamma^{2}
\end{aligned}
$$

On solving equations (1) and (2), we get

$$
(b+1) \beta^{2}-(a+1) \gamma^{2}=0
$$

Assume $\beta=x+(a+1) y$ and $\gamma=x+(b+1) y$,

it reduces to

$$
x^{2}=(a+1)(b+1) y^{2}
$$

The initial solution of the equation (5) is given by

$$
x_{0}=n^{4}+2 n^{3}-3 n^{2}-4 n-1, \quad y_{0}=1
$$

Therefore,

$$
\beta=2 n^{4}+8 n^{3}+8 n^{2}+2 n
$$

On substituting the values of $a$ and $\beta$ in equation (1), we get

$$
c=4 n^{4}+8 n^{3}+4 n^{2}-1=6 \mathrm{PT}_{2 \mathrm{n}}-\left(4 n^{3}+7 n^{2}+3 n+1\right)
$$

Hence, The triple $\left(24 \mathrm{PT}_{\mathrm{n}}, 24 \mathrm{PT}_{\mathrm{n}-2}, 6 \mathrm{PT}_{2 \mathrm{n}}-\left(4 n^{3}+7 n^{2}+3 n+1\right)\right)$ is a Dio 3-tuple with property $D(1)$.

A few numerical examples of the Dio 3-tuples satisfying the above property are mentioned below.

Table 1:

\begin{tabular}{|c|c|}
\hline$n$ & $\begin{array}{c}\text { Dio 3-tuples with property } \\
D(1)\end{array}$ \\
\hline 1 & $(24,0,15)$ \\
\hline 2 & $(120,0,143)$ \\
\hline 3 & $(360,24,575)$ \\
\hline 4 & $(840,120,1599)$ \\
\hline 5 & $(1680,360,3599)$ \\
\hline
\end{tabular}

We present below, some of the Dio 3-tuple for Pentatope number of rank mentioned above with suitable properties.

Table 2:

\begin{tabular}{|c|c|c|c|}
\hline$a$ & $b$ & $c$ & $D(n)$ \\
\hline $24 \mathrm{PT}_{n}$ & $24 \mathrm{PT}_{n}-6 \mathrm{PT}_{2 \mathrm{n}}-\left(4 n^{3}+7 n^{2}+3 n-1\right)$ & $D\left(2 n^{4}+4 n^{3}-6 n^{2}-8 n\right)$ \\
\hline $24 \mathrm{PT}_{n}$ & $24 \mathrm{PT}_{n}-6 \mathrm{PT}_{2 \mathrm{n}}-\left(4 n^{3}+7 n^{2}+3 n-3\right)$ & $D\left(4 n^{4}+8 n^{3}-12 n^{2}-16 n+1\right)$ \\
\hline $24 \mathrm{PT}_{n}$ & $24 \mathrm{PT}_{n}$ & $6 \mathrm{PT}_{2 \mathrm{n}}-\left(4 n^{3}+7 n^{2}+3 n-5\right)$ & $D\left(6 n^{4}+12 n^{3}-18 n^{2}-24 n+4\right)$ \\
\hline $24 \mathrm{PT}_{n}$ & $24 \mathrm{PT}_{n}-6 \mathrm{PT}_{2 \mathrm{n}}-\left(4 n^{3}+7 n^{2}+3 n-7\right)$ & $D\left(8 n^{4}+16 n^{3}-24 n^{2}-32 n+9\right)$ \\
\hline $24 \mathrm{PT}_{n}$ & $24 \mathrm{PT}_{n}$ & $6 \mathrm{PT}_{2 \mathrm{n}}-\left(4 n^{3}+7 n^{2}+3 n-9\right)$ & $D\left(10 n^{4}+20 n^{3}-30 n^{2}-40 n+1\right.$ \\
\hline
\end{tabular}


In general, it is noted that the triple

$$
\left(24 \mathrm{PT}_{\mathrm{n}}, 24 \mathrm{PT}_{\mathrm{n}-2}, 6 \mathrm{PT}_{2 \mathrm{n}}-\left(4 n^{3}+7 n^{2}+3 n-(2 k-1)\right)\right)
$$

is a Dio 3 -tuple with the property $D\left(2 k n^{4}+4 k n^{3}-6 k n^{2}-8 k n+(k-1)^{2}\right)$, where $k=0,1,2, \ldots$

Case 2: Construction of Dio 3-tuples for Pentatope number of rank $n$ and $n-1$.

Let $a=24 \mathrm{PT}_{n}, \quad b=24 \mathrm{PT}_{n-1}$ be Pentatope number of rank $n$ and $n-1$ respectively such that $a b+(a+b)+1$ is a perfect square say $\alpha^{2}$.

Let $c$ be any non-zero integer such that

$$
\begin{aligned}
& a c+(a+c)+1=\beta^{2} \\
& b c+(b+c)+1=\gamma^{2}
\end{aligned}
$$

Solving (6), (7) and using (4), we have

$$
x^{2}=(a+1)(b+1) y^{2}
$$

The initial solution of the equation (8) is given by

Therefore,

$$
\begin{aligned}
& x_{0}=n^{4}+4 n^{3}+3 n^{2}-2 n-1, \quad y_{0}=1 \\
& \beta=2 n^{4}+10 n^{3}+14 n^{2}+4 n
\end{aligned}
$$

On substituting the values of $a$ and $\beta$ in equation (6), we get

$$
c=4 n^{4}+16 n^{3}+16 n^{2}-1=6 \mathrm{PT}_{2 \mathrm{n}}+\left(4 n^{3}+5 n^{2}-3 n-1\right)
$$

Hence, the triple $\left(24 \mathrm{PT}_{\mathrm{n}}, 24 \mathrm{PT}_{\mathrm{n}-1}, 6 \mathrm{PT}_{2 \mathrm{n}}+\left(4 n^{3}+5 n^{2}-3 n-1\right)\right)$ is a Dio 3tuple with property $D(1)$.

A few numerical examples of the Dio 3-tuples satisfying the above property are mentioned below.

Table 3:

\begin{tabular}{|c|c|}
\hline$n$ & $\begin{array}{c}\text { Dio 3-tuples with property } \\
D(1)\end{array}$ \\
\hline 1 & $(24,0,35)$ \\
\hline 2 & $(120,24,255)$ \\
\hline 3 & $(360,120,899)$ \\
\hline 4 & $(840,360,2303)$ \\
\hline 5 & $(1680,840,4899)$ \\
\hline
\end{tabular}

We present below, some of the Dio 3-tuple for Pentatope number of rank mentioned above with suitable properties. 
G.Janaki and C.Saranya

Table 4:

\begin{tabular}{|c|c|c|c|}
\hline$a$ & $b$ & $c$ & $D(n)$ \\
\hline $24 \mathrm{PT}_{n}$ & $24 \mathrm{PT}_{n-1}$ & $6 \mathrm{PT}_{2 \mathrm{n}}+\left(4 n^{3}+5 n^{2}-3 n+1\right)$ & $D\left(2 n^{4}+8 n^{3}+6 n^{2}-4 n\right)$ \\
\hline $24 \mathrm{PT}_{n}$ & $24 \mathrm{PT}_{n-1}$ & $6 \mathrm{PT}_{2 \mathrm{n}}+\left(4 n^{3}+5 n^{2}-3 n+3\right)$ & $D\left(4 n^{4}+16 n^{3}+12 n^{2}-8 n+1\right)$ \\
\hline $24 \mathrm{PT}_{n}$ & $24 \mathrm{PT}_{n-1}$ & $6 \mathrm{PT}_{2 \mathrm{n}}+\left(4 n^{3}+5 n^{2}-3 n+5\right)$ & $D\left(6 n^{4}+24 n^{3}+18 n^{2}-12 n+4\right)$ \\
\hline $24 \mathrm{PT}_{n}$ & $24 \mathrm{PT}_{n-1}$ & $6 \mathrm{PT}_{2 \mathrm{n}}+\left(4 n^{3}+5 n^{2}-3 n+7\right)$ & $D\left(8 n^{4}+32 n^{3}+24 n^{2}-16 n+9\right)$ \\
\hline $24 \mathrm{PT}_{n}$ & $24 \mathrm{PT}_{n-1}$ & $6 \mathrm{PT}_{2 \mathrm{n}}+\left(4 n^{3}+5 n^{2}-3 n+9\right)$ & $D\left(10 n^{4}+40 n^{3}+30 n^{2}-20 n+1\right.$ \\
\hline
\end{tabular}

In general, it is noted that the triple

$\left(24 \mathrm{PT}_{\mathrm{n}}, 24 \mathrm{PT}_{\mathrm{n}-1}, 6 \mathrm{PT}_{2 \mathrm{n}}+\left(4 n^{3}+5 n^{2}-3 n+(2 k-1)\right)\right)$ is a Dio 3-tuple with the property $D\left(2 k n^{4}+8 k n^{3}+6 k n^{2}-4 k n+(k-1)^{2}\right)$, where $k=0,1,2, \ldots$

Case 3: Construction of Dio 3-tuples for Pentatope number of rank $n$ and $n-3$.

Let $a=24 \mathrm{PT}_{n}, \quad b=24 \mathrm{PT}_{n-3}$ be Pentatope number of rank $n$ and $n-3$ respectively such that $a b+(a+b)+1$ is a perfect square say $\alpha^{2}$.

Proceeding as in case1, we have the initial solution of the equation as

$$
x_{0}=n^{4}-7 n^{2}+1, \quad y_{0}=1
$$

Therefore, $\quad \beta=2 n^{4}+6 n^{3}+4 n^{2}+6 n+2$

On substituting the values of $a$ and $\beta$, we get

$$
c=4 n^{4}+8 n^{2}+3=6 \mathrm{PT}_{2 \mathrm{n}}-\left(12 n^{3}+3 n^{2}+3 n-3\right)
$$

Hence, The triple $\left(24 \mathrm{PT}_{\mathrm{n}}, 24 \mathrm{PT}_{\mathrm{n}-3}, 6 \mathrm{PT}_{2 \mathrm{n}}-\left(12 n^{3}+3 n^{2}+3 n-3\right)\right)$ is a Dio 3-tuple with property $D(1)$.

A few numerical examples of the Dio 3-tuples satisfying the above property are mentioned below.

Table 5:

\begin{tabular}{|c|c|}
\hline$n$ & Dio 3-tuples with property \\
& $D(1)$ \\
\hline 1 & $(24,0,15)$ \\
\hline 2 & $(120,0,99)$ \\
\hline 3 & $(360,0,399)$ \\
\hline 4 & $(840,24,1155)$ \\
\hline
\end{tabular}


Special Dio 3-tuples for Pentatope Number

\begin{tabular}{|l|l|}
\hline 5 & $(1680,120,2703)$ \\
\hline
\end{tabular}

We present below, some of the Dio 3-tuple for Pentatope number of rank mentioned above with suitable properties.

Table 6:

\begin{tabular}{|c|c|c|c|}
\hline$a$ & $b$ & $c$ & $D(n)$ \\
\hline $24 \mathrm{PT}_{n}$ & $24 \mathrm{PT}_{n-3}$ & $6 \mathrm{PT}_{2 \mathrm{n}}-\left(12 n^{3}+3 n^{2}+3 n-5\right)$ & $D\left(2 n^{4}-14 n^{2}+4\right)$ \\
\hline $24 \mathrm{PT}_{n}$ & $24 \mathrm{PT}_{n-3}$ & $6 \mathrm{PT}_{2 \mathrm{n}}-\left(12 n^{3}+3 n^{2}+3 n-7\right)$ & $D\left(4 n^{4}-28 n^{2}+9\right)$ \\
\hline $24 \mathrm{PT}_{n}$ & $24 \mathrm{PT}_{n-3}$ & $6 \mathrm{PT}_{2 \mathrm{n}}-\left(12 n^{3}+3 n^{2}+3 n-9\right)$ & $D\left(6 n^{4}-42 n^{2}+16\right)$ \\
\hline $24 \mathrm{PT}_{n}$ & $24 \mathrm{PT}_{n-3}$ & $6 \mathrm{PT}_{2 \mathrm{n}}-\left(12 n^{3}+3 n^{2}+3 n-11\right)$ & $D\left(8 n^{4}-56 n^{2}+25\right)$ \\
\hline $24 \mathrm{PT}_{n}$ & $24 \mathrm{PT}_{n-3}$ & $6 \mathrm{PT}_{2 \mathrm{n}}-\left(12 n^{3}+3 n^{2}+3 n-13\right)$ & $D\left(10 n^{4}-70 n^{2}+36\right)$ \\
\hline
\end{tabular}

In general, it is noted that the triple

$\left(24 \mathrm{PT}_{\mathrm{n}}, 24 \mathrm{PT}_{\mathrm{n}-3}, 6 \mathrm{PT}_{2 \mathrm{n}}-\left(12 n^{3}+3 n^{2}+3 n-(2 k+3)\right)\right)$ is a Dio 3-tuple with the property $D\left(2 k n^{4}-14 k n^{2}+(k+1)^{2}\right)$, where $k=0,1,2, \ldots$

\section{Conclusion}

In this paper we have presented a few examples of constructing a special Dio 3-tuples for Pentatope number of different ranks with suitable properties. To conclude one may search for Dio 3-tuples for other numbers with their corresponding suitable properties.

\section{REFERENCES}

1. R.D.Carmichael, History of Theory of numbers and Diophantine Analysis, Dover Publication, New york, 1959.

2. L.J.Mordell, Diophantine equations, Academic press, London, 1969.

3. T.Nagell, Introduction to Number theory, Chelsea publishing company, New york, 1981.

4. L.K.Hua, Introduction to the Theory of Numbers, Springer-Verlag, Berlin, New york, 1982.

5. O.Ore, Number theory and its History, Dover publications, New york, 1988.

6. H.John, Conway and R.K.Guy, The Book of Numbers, Springer-verlag, New york, 1995.

7. D.Wells, The penguin Dictionary of Curious and Interesting numbers, Penguin Book, 1997.

8. M.A.Gopalan, K.Geetha annd M.Somanath, On Special Diophantine Triples, Archimedes Journal of Mathematics, 4(1) (2014) 37-43.

9. M.A.Gopalan, V.Geetha and S.Vidhyalakshmi, Dio 3-tuples for Special Numbers-I, The Bulletin of Society for Mathematical Services and Standards, 10 (2014) 1-6.

10. M.A.Gopalan, K.Geetha and M.Somanath, Special Dio 3-tuples, The Bulletin of Society for Mathematical Services and Standards, 10 (2014) 22-25. 